Submitted to:

1999 Particle Acceleratớ Conference

March 29 - Ápril 2, 1999

BNL 65974

New York City, New York

\title{
MICRO \& MINI-BUNCHING OF THE SLOW EXTERNAL BEAM AT THE AGS ${ }^{*}$
}

\author{
JW Glenn', M Brennan, KA Brown BNL, D Majka YALE, A Mincer NYU
}

\begin{abstract}
The AGS's prime function when RHIC comes on line will be as a heavy ion injector, free time will exist for unique operation that can be tailored and dedicated to users. Many of the next generation of experiments require RF time structure in the spill as it is extracted to time events or assure no background during decay times. Two methods have been developed to meet these needs. Micro-bunching where the beam is forced between empty buckets producing bursts at arbitrary integer harmonics of rotation period[1], and mini-bunching where the beam is kept bunched to provide high extinction between bursts [2]. With an RF dipole the period may be increased to three times the rotation period of the beam. Further modelling and test results with beam will be presented. At high beam currents above transition, tight clusters of beam form causing severe random spike structure in the spill. Forcing the beam between empty buckets before extraction breaks up these clusters with minimal modulation of the spill at the RF frequency. Results of spill structure and loss reduction will be given.
\end{abstract}

\section{INTRODUCTION}

There are two approved future experiments that use bunched extraction from the AGS. One measuring the branching ratio of Klong to pi-zero and neutrinos needs a 250 pico-Sec burst every 300 nano-Sec. This will be accomplished using micro-bunching[1] where the debunched coasting is forced between empty longitudinal buckets by slowly reducing the main magnet field across the flat top. The extraction third integer transverse resonance is set-up at a momentum matching the RF bucket, thus the beam is extracted in short bursts at the phase where the longitudinal motion carries it to the resonance (Fig 1). Variations of this process have been modelled and measured showing that bunching can be maintained without beam loss, extraction losses can be reduced and the beam can be smoothed without major bunching. Also micro-bunching provides the possibility of fast control of the beam delivery rate. The other experiment measures the branching ratio of direct conversion of muons to electrons. Here it is vital to eliminate the possibility of any protons delivered to the pion production target during the time the muons are decaying. The process here is mini-bunching[2] where beam is kept within buckets and the bunch is slowly

\footnotetext{
Work supported in part by US Dept of Energy

- Email: glenn@bnldag.bnl.gov
}

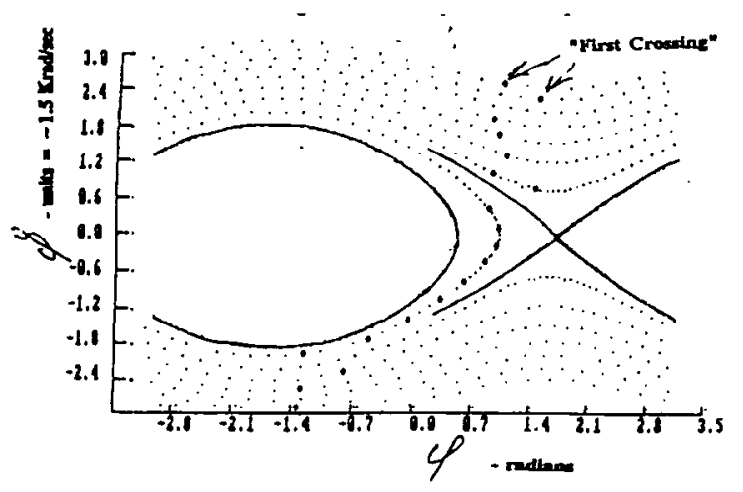

Fig 1 Particle Trajectory Near Empty Buckets.

deaccelerated to the extraction resonance. Those particles at the edge of the bunch are extracted while the space between bunches is swept of particles at the resonance turn on and thus is without beam. The option of extracting a burst every three turns has been partially tested with a kicker.

\section{FOUR DIMENSIONAL MODELLING.}

Previous modelling of micro-bunching[1] was done with separate transverse and longitudinal models. The results were then folded together. The possibility of error here prompted an effort to combine the two models and run particles in a four dimensional space of transverse $\mathrm{X} \& \mathrm{X}$ ' and longitudinal phi \& phi dot. The predicted bunch widths are similar to those previously predicted. A plot of bunch width verses the offset of the resonance momentum in units of phi dot is shown in Fig 2

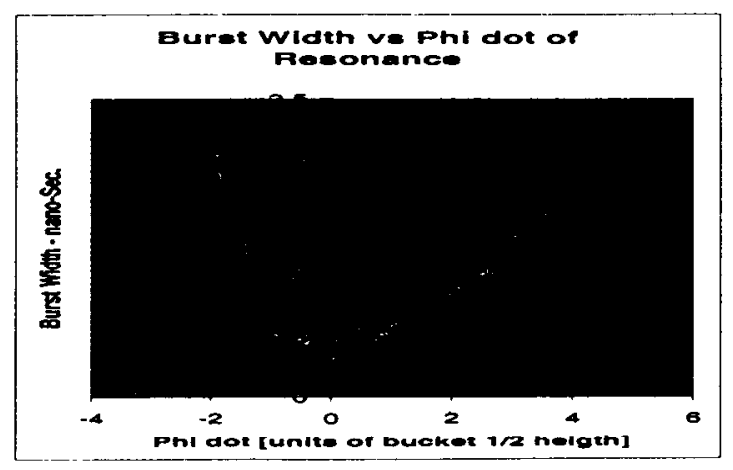

Fig 2. Burst Width as Fundtion of Monentum.

\subsection{Increased Losses during Micro-bunching}

Initial modelling of micro-bunching indicated an increase in the number of low transverse emittance particlés that would be retrapped in the stable region of phase space as the particle moves through the resonance: This has not 
been seen in any tests of micro-bunching even at full AGS intensities of $60 \mathrm{TP} / \mathrm{cycle}$. Further modelling using a more realistic distribution of particles (gausian in radius) show fewer retrapped particles as there are fewer small amplitude particles than previously estimated. Combining both transverse and longitudinal motions together show that when the resonance is before the central momentum of the RF bucket (the centre of the bucket is offset below the resonance momentum), particles spend more time near the resonance momentum than if there in no RF on. Consider particle that follows a trajectory in longitudinal phase space that approaches the unstable fixed point; it nearly stops at this point which is the lowest phi dot (highest momentum if above transition) of the trajectory. If the transverse resonance is at this momentum, the particle spends a lot of time on resonance. As Fig. 2 shows, there is some increase in bunch width.

\section{BUNCHING FACTOR.}

The results of increased RF voltage on the bunching factor was checked. At $100 \mathrm{kV}$ per turn a bunching factor (rms width divided by period of RF voltage) of 100 was measured. These tests were done during a Heavy Ion run using a gold beam. Instead of using the $93 \mathrm{MHz}$ VHF system normally used for micro-bunching, the $4 \mathrm{MHz}$ acceleration system was used. After beam was accelerated to flattop, voltage was turned down, the frequency lowered to place the buckets near the resonance with a ramp to match reducing field, then turned back on. The beam was then spiralled through the gaps between buckets. The time that a gold ion passed a counter compared to a zero crossing of the RF was recorded for each ion and a time distribution obtained and extraction efficiency noted. Runs were made for various RF voltages from 50 to 300 Volts per turn. The bunching factor for $50 \mathrm{kV}$ was 86 and for $100 \mathrm{kV}$ it was 118 . (Time distribution of the beam for $100 \mathrm{kV}$ is shown in Fig. 3.)

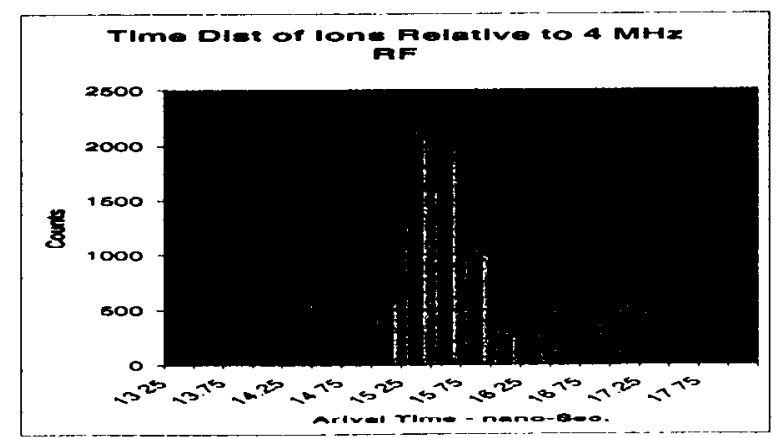

Fig 3. Burst Width for $100 \mathrm{kV}$ of RF Voltage

These factors are consistent with root voltage scaling of previous measurements with the $93 \mathrm{MHz}$ cavity. Unfortunately it was latter found for the higher voltages, the zero crossing detector saturated so timing was lost. Extraction efficiency did not drop noticeably at higher voltages in these tests indicating no increase in losses.

\section{SPILL STRUCTURE REDUCTION}

The AGS was plagued this year with random structure on the spill[3]. Total beam loss including extraction, beam splitting and transport was reduced with the VHF on line. Careful placement of the VHF buckets between the stored beam and the resonance reduces the microbunch structure in the extracted beam. It was also noted that the beam that was not extracted was trapped within the "empty" buckets and carried to the end of flat top as shown in the blowup of Fig 4 from Ref. 3 (Fig. 5). This may be a new loss mechanism associated with microbunching. An advantage of this mode of loss is that this beam may be easily dumped into a beam catcher and does not spread around the accelerator.

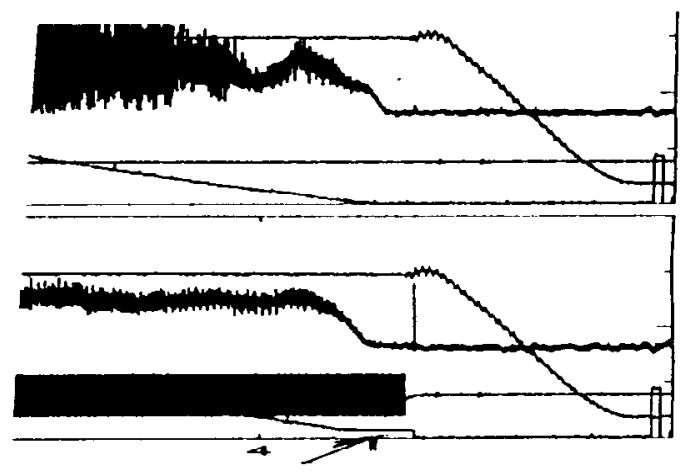

Fig 4. Beam Current (arrow) Recaptured in Homogenising Buckets.

\section{SPILL MODULATION}

In the process of setting up the VHF cavity for homogenising the beam before extraction, it was discovered that modulating the frequency of the RF strongly modulates the spill. The gain and phase response of the frequency modulation for various frequencies was measured with all slow loops closed. We measured the response of the spill to square wave modulation to estimate the dead delay of the system. A fast detector was made using the signal from an experimenters $50-100 \mathrm{MHz}$ counter into a 100 nano-Sec diode isolated integrator. The data was "eyeball fitted" assuming minimum phase response with a dead delay. The results are plotted in Fig. 5 .

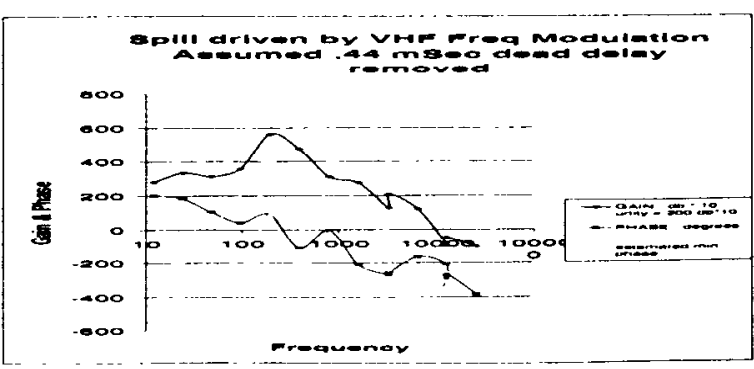

Fig 5. Gain and Phase Response of Spill to VHF Frequency Modulation. 
. The dead delay that best fits the data is only 0.4 milliSeconds which is consistent with the estimated value. The spill responds to many killo hertz of modulation.

\section{MINI-BUNCHING EXTENCTION MEASUREMENT}

We measured the cleanliness of the quiet periods between beam bursts using a separated Kaon beam looking at a production target in the Slow Beam. This was a short test run at a new extraction energy of 7 $\mathrm{GeV}$. One run showed 5 counts in the counter for a (corrected) count of 60 million during the burst for an extinction of $1.2 \times 10^{\wedge} 8$.

\section{BEAM BURST EVERY THREE TURNS.}

We did a quick check of the modelled prediction that spill structure of one third of the rotation period would be produced by introducing horizontal coherent motion during the resonant process. As no RF dipole exists for horizontal motion the tune kicker was used to create a short burst of coherent motion. The resultant spill structure was recorded using the fast detector used for spill modulation studies. The rotation period of the AGS is 2.7 uSec and the periodicity of the bursts is 8 uSec as shown on Fig. 6.

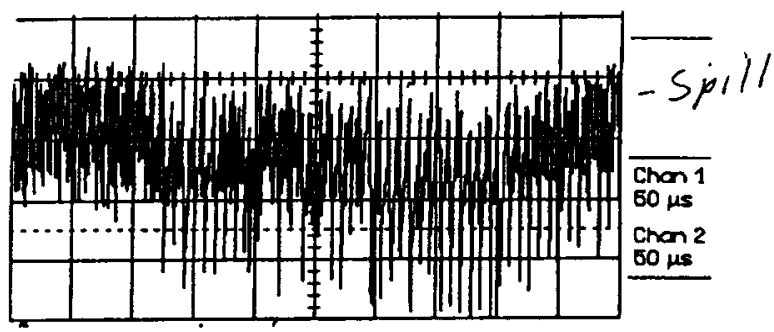

\section{REFERENCES}

[1] MICRO-BUNCHING THE AGS SLOW EXTERNAL BEAM JW Glenn, M Brennan, L Littenberg, J Rose, C Woody, A Zoltsman BNL $P$ Bergbusch, TRIUMF and Dick Mjka, YALE Session 9w005 PAC97 [2] MINI-BUNCHING THE AGS SLOW EXTERNAL BEAM JW Glenn, L Ahrens, T Hayes BNL, R Lee UC-Irvine Session 9w004 PAC97

[3] AGS RESONANT EXTRACTION WITH HIGH INTENSITH BEAMS L Ahrens, K Brown, JW Glenn, T Roser, N Tsoupas, W VanAsselt, AGS Dept, BNL Session THP140 these proceedings to be published 